\title{
Implementation of Artificial Neural Network for Mobile Movement Prediction
}

\author{
J. Venkata Subramanian ${ }^{1 *}$ and M. Abdul Karim Sadiq ${ }^{2}$ \\ 'Department of MCA, SRM University, Chennai, Tamil Nadu, India; venkat.j@ktr.srmuniv.ac.in \\ ${ }^{2}$ College of Applied Sciences, Sohar, Ministry of Higher Education, Oman; abdulkarim.soh@cas.edu.om
}

\begin{abstract}
Mobile movement estimation is an utmost requirement for proper handoff signals to the nearby mobile towers. The direction in which a mobile would move next has to be predicted so that disruption in the signal during conversation does not occur. Artificial Neural Network (ANN) with Back propagation algorithm is used for predicting the mobile movement. The results are compared with data mining approach by Go"khan (1995). The prediction performance of ANN algorithms is higher than that of the data mining approach.
\end{abstract}

Keywords: Artificial Neural Network, Back Propagation Algorithm, Data Mining, Mobile Movement, User Pattern Learning

\section{Introduction}

Mobile Movement Prediction is defined as the prediction of a mobile user's next movement in a networked environment that involves cell towers. In mobile wireless environment, a user can be able to access services while moving from one location to another. We can get the proper service availability can be achieved through proper mobile management. Mobility management in mobile computing environments covers the methods for storing and updating the location information of mobile users who are served by the system. Very little work have been done in the area of mobility prediction ${ }^{1-5}$.

Mobility management is classified into the following two components: 1) Location Management (LM) and 2) Handoff Management (HM). Location management is the process that allows the network to identify the exact location of a Mobile Terminal (MT) for a call delivery. Location management occurs in two stages: 1) Location update and 2) Call delivery to the user ${ }^{6}$. When a mobile terminal moves from one Location Area (LA) to another it performs a location update procedure that provides the network with its location information. On the other hand, call delivery refers to querying network for location information on the called MT. In order to deliver the call, the network needs to identify the exact location of the called MT using a paging procedure ${ }^{6}$. The goal of this procedure is to find the called MT's current cell, within its registered.

Location management methods are divided into two major components ${ }^{7}$ : storage-based and non-storagebased methods. The first group includes methods based on learning processes, which require knowledge of mobile user behavior. The second group includes methods based on specific algorithms and network architectures. In this paper, we have discussed and implemented the Artificial Neural Network, belong to first group ${ }^{8}$.

We are going to introduce the Home Location Register (HLR) and Visitor Location Register (VLR) for location update and call delivery procedures established standards are available ${ }^{9}$. The HLR for a given network contains the network's subscriber profiles, while a VLR stores the profiles of the users that are currently roaming within Location Area (Las) associated with that specific VLR.

In mobile wireless networks, almost all users follow regular routines during working days, residing mostly at their place of work. For these users, it is easy to predict with perfect accuracy their location at a particular time of

${ }^{*}$ Author for correspondence 
day. The main aim of user pattern learning location prediction schemes is to leverage off this information to reduce location and paging requests. There are three different types of Users, depending on the predictability of their daily routine: highly predictable users (high probability), significantly predictable users (medium level probability), and un predictable users (easily cant get the moments) ${ }^{6}$. This differentiation is similar to classification given ${ }^{10}$.

The User Pattern Learning (UPL) approach includes with each user a list of location areas (LA) where the mobile user must be located within a given time interval. When a call arrives for an MT, each location within the list is paged sequentially until the MT is found. When a user moves between locations within the list, no location update is required. The list is stored at an Intermediate Location Database (ILD) ie., temporary database ${ }^{6}$, associated with a Mobile Switching Centers (MSC) as well as within the user's MT. Based on the movement of the user, the cost reduction carried. We assume that, when the user follows their expected routine, the location update cost can be reduced.

If the position of a mobile user is always known in advance, then, no need of external registration. Thus, the optimal location area is given by a single cell, which, in turn, minimizes paging costs ${ }^{11}$. User mobility information can be used for mobility management (traffic routing), to administrate and maintain the following network resources they are, resource allocation, call admission control, congestion, and flow control and to analyze handoff algorithms in integrated wired/wireless networks ${ }^{12}$. User mobility patterns can also be useful for system recovery ${ }^{13}$. By means of a fuzzy logic algorithm, a users' location is forecast by the system, which eliminates the need of a backup. By this way, users will not experience delays.

\section{Artificial Neural Networks}

In this paper, to learn the regular behavior and routines, we are using the Artificial Neural Network ANN). Pattern classification (from the area of Pattern Recognition) can be explained as the problem of labeling test patterns derived by a particular application domain. A classification system may be trained by a set of data features. They prepared well adequately or not.

Generally, ANN systems are capable of "learning" from the given data set and creating input-output relationships based on a "test" set of data strictly. It is desirable for the "test" data that the system "learns" from to be as representative of the complete data set as possible; trends not seen in the test data set will not be "learned" by the neural network system. Once training was over, now the network is ready for application. For satisfactory application, it is necessary that the training data contain input sets (and the associated output values) that represent the entire range of possible future inputs; the system will only perform as well as it has been trained ${ }^{8}$. Some time, the training examples may contains errors also.

The fundamental trait of intelligence is the capability of learning. This learning process in the ANN context can be viewed as the problem of updating network architecture and connection weights so that a network can efficiently perform a particular work. The network learns the connection weights from available training patterns $s^{6,8}$. Iteratively updating the weights in the network then the performance can be improved easily. ANN's ability to automatically learn from examples makes them attractive $^{6}$. ANNs learns underlying rules (like input-output relationships) from the given collection of representative examples. This is one of the major advantages of Artificial Neural networks over traditional expert systems.

Neural networks adopted from their computing power through their ability to learn and then generalize; generalization refers to the capability of the neural network to produce reasonable outputs for inputs not encountered during training. Due to this suitable reason, ANN has been used to predict the movement of mobile users so that predicting the position of a user in advance is possible and reduce the paging cost based on the predicted the next movement to destination also. The impact on the performance of location management with ANN is minimized.

The cost of the UPL is classified into four components: training task, maintenance and update of the user's profile, location update, and call delivery. Although ANN learning times are relatively long, evaluating the learned network in order to apply it to a subsequent instance (maintenance and update of the user's profile, location update and call delivery) must be done in very fast. In ANN, performance is improved over time by iteratively updating the weights in the network. This paper proposes a User Pattern Learning strategy that reduces the signaling cost of a location update by increasing the intelligence in the location procedure. 
The user pattern learning process associated to with each and every user. The users are classified into three different categories depending the predictability of their daily routine: users who have a very high probability of being where the system expects them to be (highly predictable users), users who have a certain likelihood of being where the system expects them to be (quasi-predictable users), and users whose position at a given moment is undetermined (randomized users) ${ }^{8}$. The predictability of highly predictable and quasi-predictable users can be used by the system to reduce the number of location update operations. So, after the learning process completes, we get the mobile user's behavior associated with known location areas. Then, a profile is built for the mobile user (Table 1). When a call arrives for a mobile, the call is stored in terms of pages and stored sequentially in the location. When a user moves between location areas in this list, no location updates are needed. The cost reduction depends on the behavior of each class of user. We can assume, when the user moves his / her expected behavior, the cost of location update is reduced, even if accesses to HLR are minimized when calls are received from relatively close areas.

The ANN topology is trained with different types of weight updating algorithms. They are called supervised methods and unsupervised methods. In supervised method, both the inputs and outputs are considered for movement prediction. On the other hand, the unsupervised method, only the inputs of a pattern are considered. The output obtained from the input to the output layer is compared with the desired output of the pattern. This difference between the calculated output of the network and the desired output is called Mean squared error (MSE) ${ }^{6}$ of the network.

The error is propagated in backwards, such that the weights connecting the different layers are updated. By this process the MSE of the network for the pattern presented is minimized. This procedure is a cumulative work and summed ${ }^{8}$. After presenting the last training pattern, the network is considered to have learnt all the training patterns through iterations, but the MSE is large. To minimize MSE, the network has to be presented with all the training patterns many times. There is no guarantee that the network will reach the minimum. Instead it will reach one of the local minima. The MSE may increase some times, which means divergence rather than convergence. Sometimes, there may be some variances between convergence and divergence.

We can stop the training of the network, when either by considering MSE or by considering classification performance as the criterion. When classification performance is considered as the criterion, test patterns are noted at the end of iteration. Once the desired performance is obtained, training of the network is stopped. When MSE is considered as the criterion, one may not know the exact MSE to which the network has to be trained. If the network is trained till it reaches a very low MSE, over-fitting of the network occurs. Over-fitting represents the loss of generality of the network. That is the network classifies only the patterns which are used during training and not the test patterns $s^{6,8}$.

The movement of mobile terminals MT are predicted in various algorithms, The Gaussian Mixture model ${ }^{11}$ method highly depends on the choice of training data and thus the method is yet to be studied extensively in real data. Next is Bayesian Learning Method ${ }^{12}$. It is a traditional method for topology independent user tracking. Geographical coverage and streets network are not considered.

In Markovain Approach Algorithm ${ }^{13}$, is not having a capability of accurate prediction of user movements in case of uniform movement distributions. This model named Random Walk Model ${ }^{13}$ does not include the user's motion history, the states that were visited in the past.

In $\mathrm{KMPM}$ algorithm ${ }^{14}$, predicted the next location of mobile user in a mobile web computing system using Knowledge grid. But it is for distributed system.

Movement of mobile nodes were predicted by Pratim ${ }^{15}$. But random movement pattern by the user were not tested and the expected prediction accuracy was not obtained.

A two step method was suggested by Velmurugan ${ }^{16}$. The first step to derive a mathematical model for mobility rules extraction and the second step involves clustering

Table 1. Example of a user's behavioral profile

\begin{tabular}{lllllll}
\hline Serial & Profile & Mobile & Local area & Expected \\
Number & Number & $\begin{array}{l}\text { Timestamp } \\
\text { Switching } \\
\text { Centers } \\
\text { Identification }\end{array}$ & & Identification & $\begin{array}{l}\text { Number } \\
\text { (Explicit }\end{array}$ & of visits \\
& & & Local user) & \\
\hline
\end{tabular}


of extracted rules. In this method the accuracy is around $84 \%$ only.

There is no method presented to discover these mobility patterns in Global Mobility Model and Local Mobility Model. When the random movements of the user increases Automatically, the performance of MMP decreases in Mobile Motion Prediction method.

\section{Back Propagation Algorithm}

A new improvement of Back Propagation Neural Network learning algorithms with Adaptive gain has been done already. This BPA uses the steepest-descent method to reach a global minimum ${ }^{17}$. Figure 1 presents flow-chart describing the working of BPA

First the no. of layers and no. of nodes are counted. Initialize the weights for the first iteration. Patterns are studied and calculate the observed outputs of nodes in the subsequent layers. From input to output layer the outputs are calculated. Update the weights between the different layers. In the next step, Checked and updated of weights for all layers, if yes, Calculate the Mean Squared error for all the patterns. This was applied for all the patterns.

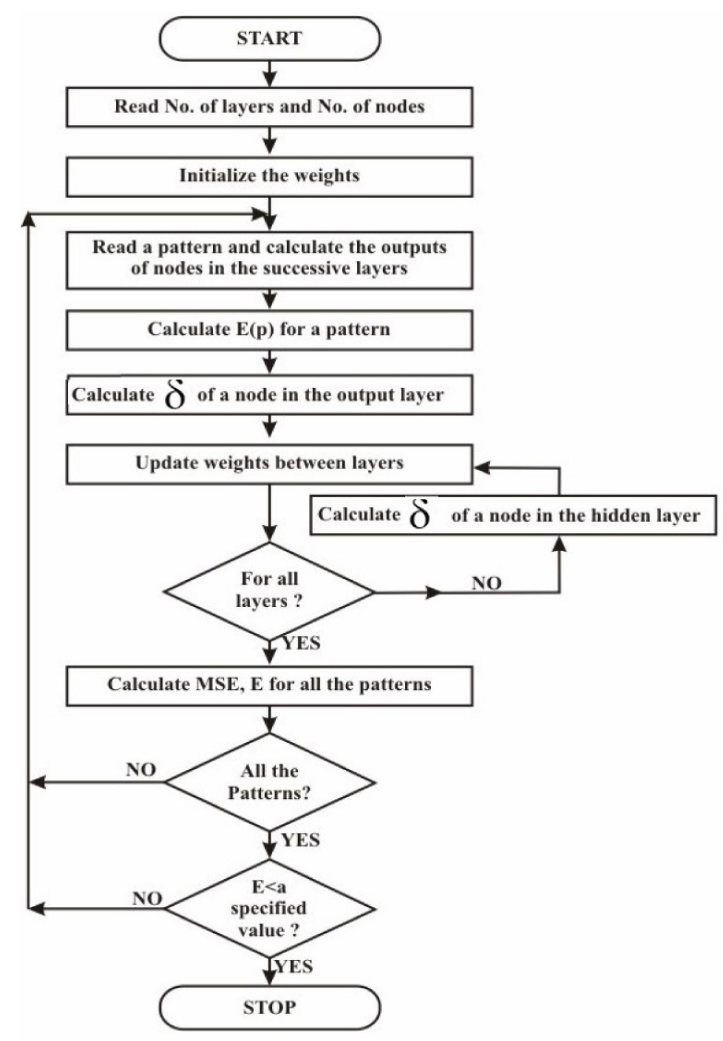

Figure 1. Flow chart of the back propagation algorithm.
Check the specified value E $>$ a if yes, we can stop the process. if not, the backward propagation should be carried out ${ }^{6,8}$.

\section{Steps involved in training $\mathbf{B P A}^{8,17}$}

Forward propagation:

Step 1: ANN weights are initialized.

Step 2: The inputs and outputs of training patterns are presented to the BPA network. The output of each node in the successive layers is calculated using following equation (1)

$$
\mathrm{o}_{\text {(output of a node) }}=1 /\left(1+\exp \left(-\sum \mathrm{W}_{\mathrm{ij}} \mathrm{X}_{\mathrm{i}}\right)\right)
$$

Step 3: Using equation given below the error of the pattern calculated (2).

$$
\mathrm{E}(\mathrm{p})=(1 / 2) \sum(\mathrm{d}(\mathrm{p})-\mathrm{o}(\mathrm{p}))^{2}
$$

Backward propagation (Weight updation)

Step 4: The error for the nodes in the output layer is calculated using following equation (3).

$\delta_{\text {(output layer) }}=\mathrm{o}(1-\mathrm{o})(\mathrm{d}-\mathrm{o})$

Step 5: The weights from output layer to hidden layer are updated using equation (4).

$\mathrm{W}_{(\mathrm{n}+1)}=\mathrm{W}_{(\mathrm{n})}+\eta \delta_{\text {(output layer) }} \mathrm{o}_{\text {(hidden layer) }}$

Step 6: The error for the nodes in the hidden layer is calculated using equation (5)

$\delta_{\text {(Hidden layer) }}=\mathrm{o}(1-\mathrm{o}) \sum \delta_{\text {(output layer) }} \mathrm{W}_{\text {(updated }}$
weights between hidden and output layer)

Step 7: The weights between hidden layer(s) and input layer are updated using equation

$\mathrm{W}_{(\mathrm{n}+1)}=\mathrm{W}_{(\mathrm{n})}+\eta \delta_{\text {(hidden layer) }} \mathrm{o}_{\text {(input layer) }}$

From the above steps one iteration was completed. The Second pattern is presented for the above steps are followed for the second weight updation. When all the training patterns (iterations) are completed, a cycle of iteration or task is completed. The errors of all the training patterns are calculated using equation $(7)^{6,8}$.

$\mathrm{E}(\mathrm{MSE})=\sum \mathrm{E}(\mathrm{p})$

\section{Outcome of BPA}

Here, the topology of ANN used the no. of nodes in the input layer is 2, the no. of nodes in the hidden layer is 10 and the no. of nodes in the output layer is 1 . The labeling 
is a number allotted for the present location. It is mandatory to use huge amount of patterns to be presented for training ANN.

Here, only 1000 patterns have been considered for training purpose ${ }^{18}$ because, it would take enormous amount of time for the ANN to learn the patterns. The dataset has been separated from training and testing. Training indicates the formation of final weights which indicate a thorough learning of movement of mobile users.

Figure 2 shows the convergence curve for the topology of 2 nodes to 10 nodes in the hidden layer. It converged in around 405 iterations. If the number of patterns is further increased from 1000 then the convergence iterations will increase. In addition, the convergence depends on the orthogonality of data presented for training. If the subsequent patterns are not orthogonal, the convergence would take a long time or it may not converge.

The percentage of user movement prediction is presented for various nodes in the hidden layer (Figure 3).

The obtained percentages of prediction for the testing patterns calculated from all training iterations were presented. The percentage of prediction is high in less iteration with only 10 nodes in the hidden layer.

\section{Conclusion}

In this paper, we have presented the user pattern learning approach $^{6}$ by using neural networks to reduce location

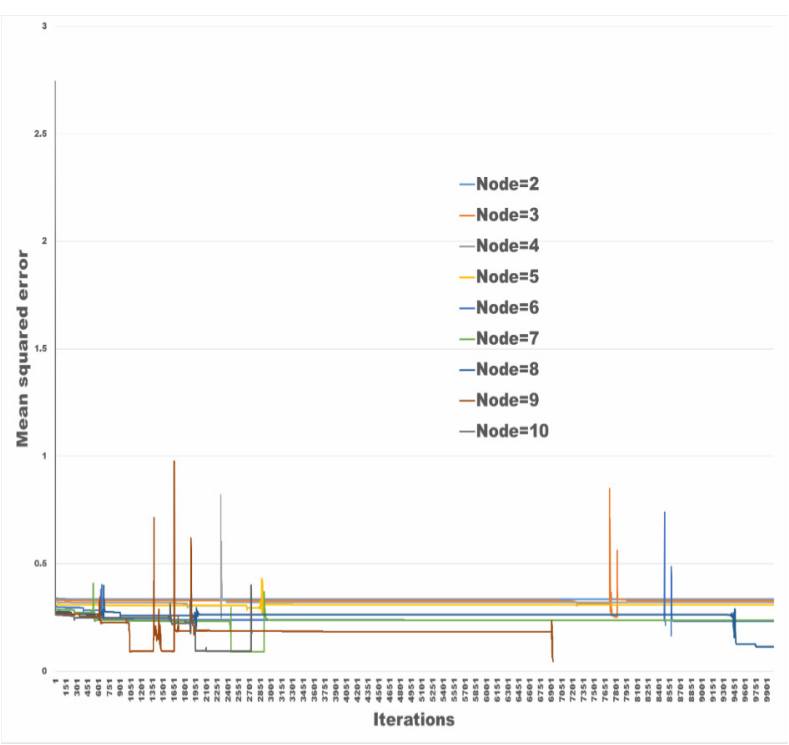

Figure 2. Mean Squared Error of the network trained by using Back propagation algorithm for the location prediction.

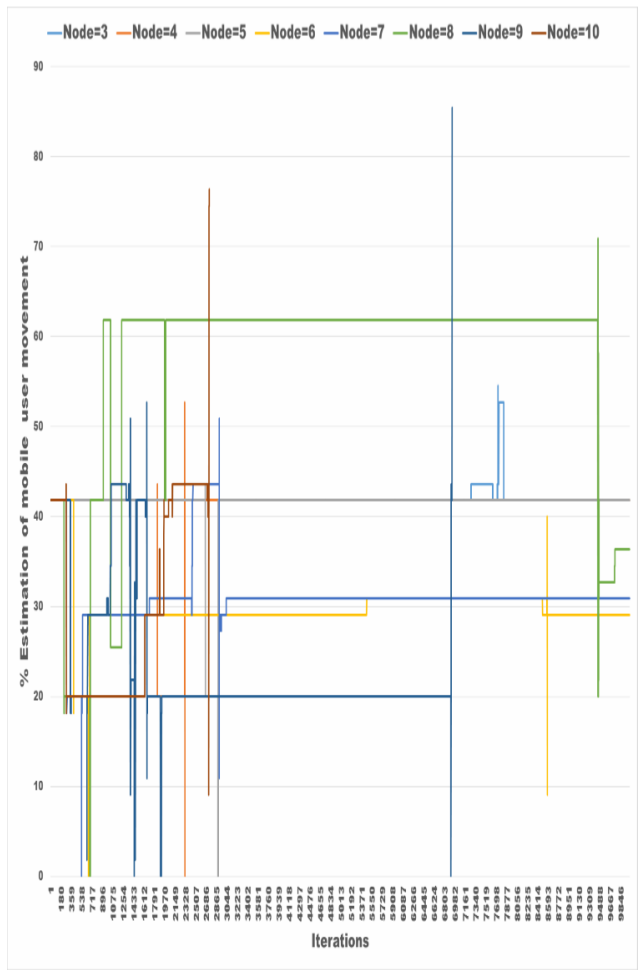

Figure 3. Percentage of user movement prediction by BPA.

update signaling cost by increasing the intelligence of the location procedure. This approach associates to each user a list of cells where mobile is likely to be with a given probability in each time interval. The list is ranked between the most likely and the least likely place where a user may be found. When a call arrives for a mobile, it is paged sequentially in each location within the list. When a user moves between location areas in the list, no location updates are needed. The performance evaluation results confirm the efficiency and the effectiveness of user pattern learning approach $^{6,8}$. This desired improvement represents a large reduction in location update and paging signaling costs.

\section{References}

1. Wu HK., M-H Jin, Horng J-T, Ke C-Y. Personal paging area design based on mobiles moving behaviors. Proceedings of the IEEE Conference on Computer and Communications (IEEE INFOCOM2001); 2001. p. 21-30.

2. Rajagopal S, Srinivasan RB, Narayan RB, Petit XBC. GPSbased predictive resource allocation in cellural networks. Proceedings of the IEEE International Conference on Networks (IEEE ICON02); 2002. p. 229-34.

3. Liu GY, Gerald MQ. A predictive mobility management algorithm for wireless mobile computing and 
communications. Proceedings of the IEEE International Conference on Universal Personal Communications; 1995. p. $268-72$.

4. Liu T, Bahl P, Chlamtac I. Mobility modeling, location tracking, and trajectory prediction in wireless ATM networks. IEEE J Select Area Commun. 1998; 16(6):922-36.

5. Aljadhai A., Znati T. Predictive mobility support for QoS provisioning in mobile wireless environments. IEEE J Select Area Commun. 2001; 19(10):1915-30.

6. Singh AP, Karnan M. A dynamic location management scheme for wireless networks using cascaded correlation neural network. International Journal of Computer theory and Engineering. 2010;2(4):581-85.

7. Tabbane S. Location management methods for 3rd generation mobile systems. IEEE Comm Magazine. 1997; 35(8):72-8.

8. Singh AP, Karnan M. Using a novel intelligent location management strategy in wireless networks. International Journal of Computer and Network Security. 2009; 1(1):9 -15 .

9. Suh B, Choi J, Kim J. Design and performance analysis of hierarchical location management strategies for wireless mobile communication systems. Computer Comm. 2000; 23(5):550-60.

10. Pollini G, Chih-Lin I. A profile-based location strategy and its performance. IEEE J Selected Areas in Comm. 1997; 15(8):1415-24.

11. Thann An N, Phuog T M. A Gaussian mixture model for mobile location prediction. IEEE; 2007. p. 152-57.
12. Akoush S, Sameh A. Movement prediction using bayesian learning for neural networks. IEEE. (ICSNC 2007).

13. Fulop P, Szabo S, Szalka T. Location prediction methods with markovain approach and extended random walk model. IEEE Computer Society; 2007.

14. Sakthi U, Bhuvaneswaran RS. Mobility prediction of mobile users in mobile environment using knowledge grid. International Journal of Computer Science and Network Security. 2009; 9(1):303-9.

15. Bhattacharya PP, Bhattacharya M. Artificial neural network based node location prediction for applications in mobile communication. International Journal of Computer Applications in Engineering Sciences. 2011; I(II):104-7.

16. Velmurugan L, Thangaraj P. Mobility prediction using hidden genetic layer based neural network. Life Science Journal. 2013; 10(4s):549-53.

17. Austin N, Kumar PS, Purushothaman S. Mixed refrigerants suitability analysis using artificial neural networks. Journal of Engineering and Applied Sciences. 2012; 7(5):588-92.

18. Sujatha P, Purushothaman.S, Rajeswari R. Detecting the presence of hidden information using back propagation neural network classifier. International Journal of Computer Science and Information Security. 2012; 10(12):36-41.

19. Singh AP, Karnan M. Intelligent location Management for UMTS Networks using fuzzy neural networks. Journal of Engineering and Technology. 2010; 2(1):1-12. 